\title{
环糊精及其衍生物催化的有机反应
}

\author{
洪诗斌 $a, b$ 刘梦艳 ${ }^{a, b}$ 张 薇 ${ }^{a, b}$ 邓 维 $*, a, b$ \\ ( ${ }^{a}$ 上海大学纳米科学与技术研究中心 上海 200444) \\ $\left({ }^{b}\right.$ 上海应用技术学院化工学院 上海 201418)
}

\begin{abstract}
摘要 环糊精及其衍生物能将有机小分子等包合进疏水空腔, 改变有机小分子在水中的溶解性, 还能通过电荷作用影 响分子的电性环境. 环糊精还是天然的手性分子, 将环糊精及其衍生物用于催化有机反应不仅能使反应在温和的条件 下得到良好的收率, 还能运用于不对称合成. 本文就近年来环糊精催化的有机反应分五部分概述: 非修饰环糊精、金属 离子参与、无金属离子参与催化的有机反应以及它们在手性催化、光化学反应的运用.
\end{abstract}

关键词＼cjkstart环糊精; 超分子; 有机合成; 催化反应; 不对称合成

\section{Organic Reactions Catalyzed by Cyclodextrin and Its Derivatives}

\author{
Hong, Shibin ${ }^{a, b}$ Liu, Mengyan ${ }^{a, b}$ Zhang, Wei ${ }^{a, b}$ Deng, Wei*a, \\ ( ${ }^{a}$ Research Center of Nano Science and Technology, Shanghai University, Shanghai 200444) \\ ( ${ }^{b}$ School of Chemical and Engineering, Shanghai Institute of Technology, Shanghai 201418)
}

\begin{abstract}
Cyclodextrin (CD) and its derivatives catalyzed organic reactions, including asymmetric reactions, show mild conditions with good yield. This review is an overview of recent advances of CD catalyzed reactions, which is organized in the order of the following reaction types: the modified cyclodextrin catalyzed organic reaction, cyclodextrin catalyzed organic reaction of metal ion present, cyclodextrin catalyzed organic reaction without metal ion, and cyclodextrin catalyzed organic reactions in application of asymmetric synthesis and photochemical reactions.

Keywords cyclodextrin; supramolecule; organic synthesis; catalyst reactions; asymmetric synthesis
\end{abstract}

超分子体系广泛地存在于自然界中, 为多个分子间 通过非键合作用聚集在一起的分子集合体，其协同地实 现了生物体的所有生命功能. 1987 年的诺贝尔奖就是表 彰了 Cram, Pedersen 和 Lehn 对超分子的研究, Lehn 首次 提出了超分子的概念—— “超越分子的多个分子”。不 少超分子催化体系被设计合成, 以对生物体的化学反应 进行机理研究, 应用于绿色高效的不对称合成中. 多种 超分子体系均被应用于催化反应中, 包括冠醚、环糊精、 杯芳烃、葫芦腿和树枝形聚合物等. 冠醚与中性分子、 离子形成主客体络合物, 改变了客体分子的化学活性, 从而实现选择性催化 ${ }^{[1]}$. 调节杯芳烃的空腔自由度, 可 以有效地形成双金属核络合物的催化体系 ${ }^{[2]}$. 葫芦嫝的 特殊结构使得它具有离子偶极、疏水等作用, 它在分子
反应器方面有良好的催化应用 ${ }^{[3]}$. 树枝形聚合物具有精 确的纳米构造, 其精确的层数与体积可由合成步骤决 定 ${ }^{[4]}$. 环糊精便宜易得、对生命体无毒、可被生物降解, 具有优良的分子识别性、良好的生物相容性、相转移功 能, 且环糊精易于衍生化, 具有很好的催化潜能. 因此 被广泛地应用于构建化学催化剂, 进行高效、选择性且 对环境友好的绿色化学反应 ${ }^{[5]}$.

环糊精 $(\mathrm{CD})$ 是由多个 $\mathrm{D}(+)$-葡萄糖单元通过 $\alpha-1,4-$ 糖苷键首尾相连而成的环状化合物. 常见的环糊精有 $\alpha-, \beta$-和 $\gamma-\mathrm{CD}$, 分别由 $6 \sim 8$ 个葡萄糖单元组成. 其中 $\beta-\mathrm{CD}$ 作为最廉价的环糊精且具有多种独特的理化性质, 成为目前研究最热门的环糊精. 环糊精的空间结构如图 1 所示, $\mathrm{CD}$ 的空间结构呈倒桶状, 圆筒内的各个葡萄糖

* E-mail: dengwlcq@ustc.edu

Received September 2, 2014; revised October 19, 2014; published online November 5, 2014.

Project supported by the Eastern Scholar, Shanghai Pujiang Program, the Key Subject of Shanghai Municipal Education Commission (No. J50704), and the National Natural Science Foundation of China (Nos. 21102088, 21174081).

上海市东方学者、上海市浦江学者、上海市教育委员会重点学科建设(No. J50704)和国家自然科学基金(Nos. 21102088, 21174081)资助项目. 


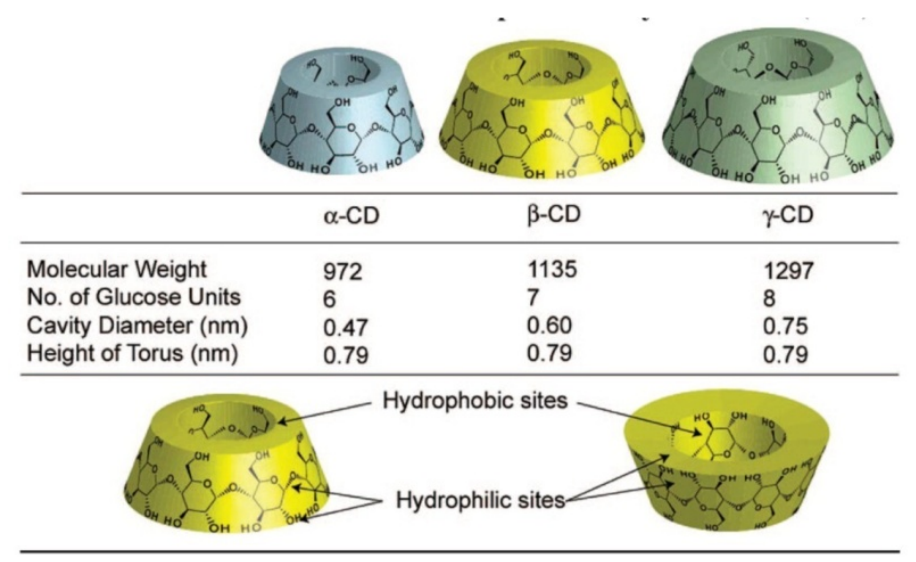

图 1 环糊精结构图

Figure 1 Structure of cyclodextrin

单元皆处于椅式构象. 环糊精外侧含有大量的差基, $\alpha$-, $\beta$-和 $\gamma-\mathrm{CD}$ 在 C-2、C $-3 、 \mathrm{C}-6$ 含有的羟基的个数上分别 为各 $6 、 7 、 8$ 个. 这些羟基都在环糊精的外侧, 使得环 糊精具有较好的亲水性. 因为环糊精内部不具有羟基, 所有环糊精具有独特的疏水空腔, 虽然在环糊精空腔中 的一圈糖苷键上有 $n$ 个类醚氧原子, 但由于它们处于空 腔内侧 $\mathrm{C}-\mathrm{H}(3)$ 和 $\mathrm{C}-\mathrm{H}(5)$ 构成的两圈共 $2 n$ 个 $\mathrm{C}-\mathrm{H}$ 键 的屏蔽之下，使其疏水作用更加明显. 正是由于 CD 具 有 “内疏水、外亲水” 的特殊空间结构, 使得环糊精得 到广泛的应用.

环糊精的空腔可以和有机小分子形成主客体包合 物, 催化有机反应顺利进行, 且多数反应可以在水相中 进行; 另外，环糊精具有分子体积大，位阻效应明显的 特性, 可以使得有机反应具有区域选择性; 环糊精还是 天然的手性分子，在手性催化反应中有更多应用; 同时 环糊精作为人体内的一种生物酶, 能够催化许多复杂的 有机反应, 加之无毒又廉价, 使环糊精催化有机反应可 以工业化、产业化.

非修饰的环糊精可以和疏水性的小分子形成包合 物, 提高底物在水中的溶解度, 因而常被作为相转移催 化剂, 应用于水相中的有机反应中. 但由于其在有机溶 剂中溶解性差、有限的疏水区域和催化活性, 使得环糊 精在应用上受到局限. 对环糊精进行化学修饰可以很好 的弥补环糊精的不足. 可以构建有新结构和新功能的超 分子体系, 设计合成具有多种复合功能的材料, 有效地 扩展环糊精的应用范围. 经过多年的研究, 对于环糊精 的修饰技术已经日趋完善，可以实现对环糊精 C-2, C-3 和 C-6 位羟基选择性地进行修饰以及全羟基修饰 ${ }^{[6]}$. 环 糊精因为其分子识别性而被最早也是最广泛被应用为 生命体催化剂——酶的模拟中, 在环糊精的差基直接引 入咪唑基可以直接模拟水解酶的功能，对乙酸对硝基酚
酯具有明显的催化水解活性，同时其二元取代物对核糖 核酸酶的水解表现了高度立体选择性 ${ }^{[7]}$. 在生物化学中, 环糊精同样得到了很好的应用, $\mathrm{Liu}$ 等 ${ }^{[8]}$ 将富勒烯 $\mathrm{C}_{60}$ 连 接到环糊精上, 在水溶液里得到了很好的光驱动 DNA 切割结果.

环糊精参与的催化反应受到了人们越来越多的重 视，环糊精在有机催化以及高分子合成等方面的研究 被大量地报道，尤其最近几年，环糊精在过渡金属催化 和不对称合成等方面取得了不少进展. 本文首先基于传 统的相转移催化剂, 综述了非修饰的环糊精催化有机反 应; 然后对环糊精衍生物催化的有机反应分为两部分概 述一一有金属离子参与和无金属离子参与; 最后对环糊 精衍生物应用于手性催化反应和环糊精及其衍生物应 用于光化学反应进行了介绍.

\section{1 非修饰的环糊精催化的有机反应}

非修饰的环糊精具有疏水空腔以及较好的水溶性, 可以与疏水有机小分子相互作用，形成可逆的主客体包 合物，提高疏水有机小分子在水中的溶解性，使有机反 应可以在水相中进行。同时环糊精上具有大量的羟基, 能够与有机小分子通过分子间作用力形成氢键, 影响有 机分子的电性环境，使反应有良好的收率.

Rao 等 ${ }^{[9]}$ 报道了一系列的使用非修饰环糊精催化的 有机水相反应. 环糊精具有的疏水空腔可与有机小分子 形成包合物，增加了分子间的碰撞几率，使得反应可以 快速高效地进行. Rao 等 ${ }^{[9 a]}$ 使用非修饰的环糊精催化螺 环吲哚的合成(Eq. 1), 反应为多组分水相反应. 在不加 环糊精时，即使反应时间延长至 $24 \mathrm{~h}$ ，反应产率也不足 $20 \%$. 在加入 CD 后，反应在温和的条件下可以得到很 好的收率. Rao 通过核磁确定了反应的机理. 首先吲哚 醌进入环糊精的空腔与环糊精形成了包合物，包合物内 
环糊精的羟基与吲哚醌发生电荷效应, 形成的氢键促进 反应的加速进行.. Rao 课题组 ${ }^{[9 b]}$ 将非修饰环糊精在温和 的条件下催化吲哚与硝基烯烃发生傅克烷基化反应 (Scheme 1), 反应过程是环糊精先将吲哚以及硝基烯烃 包合进入空腔, 增加反应物在水中的溶解性, 在电荷性 等因素下, 环糊精上羟基可促进反应物发生傅克烷基 化. 而且非修饰环糊精能够催化环氧化物以及氮杂环丙 烷具有区域选择性的亲核开环反应(Scheme 2) ${ }^{[9 \mathrm{c}]}$. 反应 过程也是利用环糊精的空腔以及其大量羟基的氢键作 用, 并且由于环糊精的空腔有大小口之分, 反应物进入
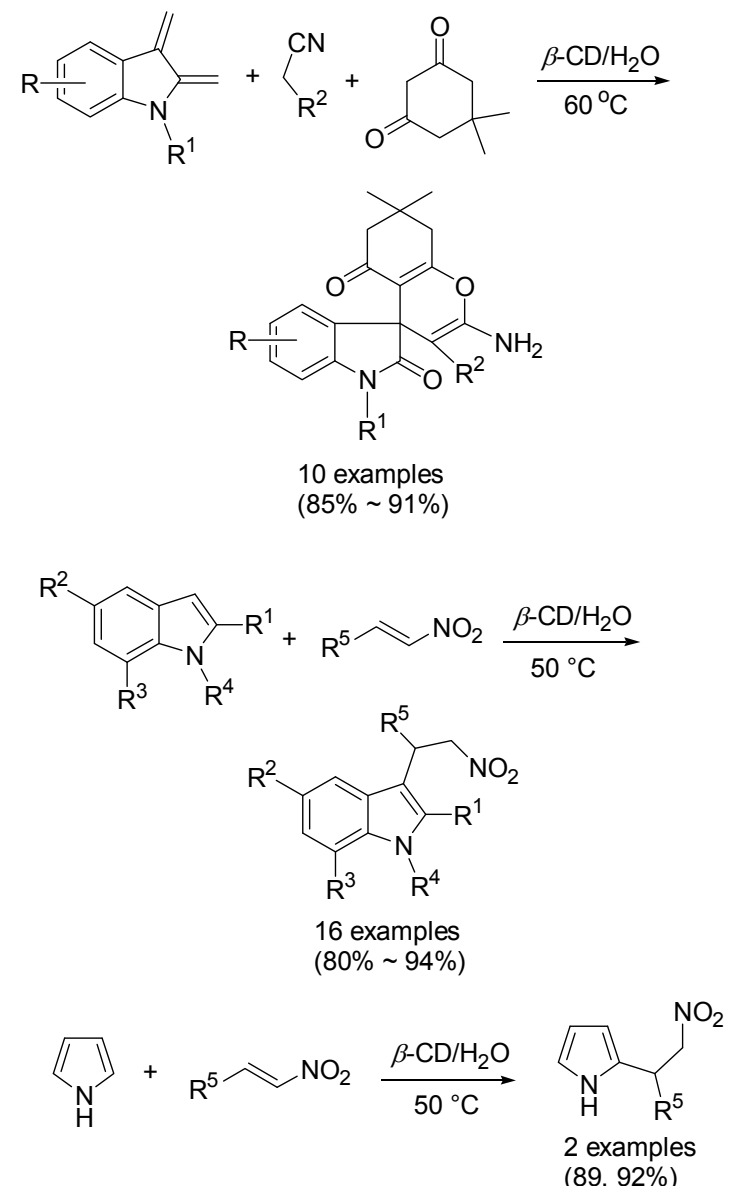

图式 1 环糊精催化付克烷基化反应

Scheme 1 Friedel-Crafts alkylation reaction catalyzed by CD

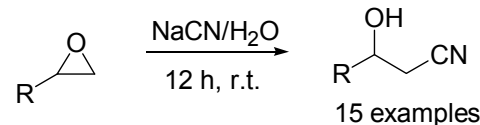

$$
\begin{aligned}
& \text { (77\% 90\%) }
\end{aligned}
$$

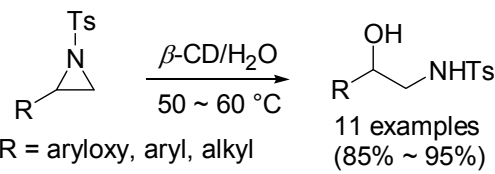

图式 2 环糊精催化环氧化物、氮杂环丙烷发生亲核开环反应 Scheme 2 Nucleophilic ring-opening reaction catalyzed by CD
环糊精会受到刚性空腔的影响，环氧化物的开环倾向于 没有取代基的一端，这是因为环糊精体积比较，有较好 的位阻效应，使得反应产物具有区域选择性.

Nageswar 等 ${ }^{[10]}$ 进一步对非修饰的环糊精催化有机 反应进行了深入研究. 在合成喹唑啉及其衍生物的反应 中(Eq. 2), 在室温且未添加环糊精时，产率只有 55\%; 在加入 $10 \mathrm{~mol} \%$ 环糊精以及温度在 $55 \sim 60{ }^{\circ} \mathrm{C}$ 时, 反应 产率可高达 $86 \%$ 以上. 而且通过核磁确定了环糊精与反 应物形成了包合物，其反应机理是由环糊精包合有机小 分子, 增加苯甲醛和苯胺在水中的溶解性而促进反应加 快进行. 最近，他们发现了一个在中性条件下就可以获 得良好收率的水相反应——环糊精催化吲哚醌与氨基 脲/氨基硫艮加成反应(Scheme 3). 由于吲哚醌在水中溶 解度差, 即使在较高的反应温度下, 目标产物的收率也 很低. 在一样的反应条件下, 室温加入 $\beta-\mathrm{CD}$ 可以得到 $55 \%$ 中等产率. 将温度提高到 $50 \sim 55{ }^{\circ} \mathrm{C}$, 反应产率可 以大大提高到 $89 \%$. Nageswar 还对比了 $\alpha-, \beta$-和 $\gamma-\mathrm{CD}$ 三 种未修饰环糊精对反应的影响, 实验结果表明, 只有在 使用 $\beta$-CD 作为超分子催化剂时, 反应收率是最高的. $\beta-\mathrm{CD}$ 在实验结束后可以回收再利用, 其催化活性几乎 没有降低. Nageswar 等还报道了一个温和、高效合成噻 唑、硒唑的新方法, 反应实现了一锅法水相反应. 当加 入䇻选出的最佳的环糊精添加量 $10 \mathrm{~mol} \% \mathrm{CD}$, 反应产 率可以从 $45 \%$ 上升到 $70 \%$ 左右. 这是首例报道三组分反 应将苯乙炔、NBS、硫嫝/硒脲一锅法合成噻唑/硒唑, 以 苯乙炔为原料合成噻唑、硒唑及其衍生物(Scheme 4).

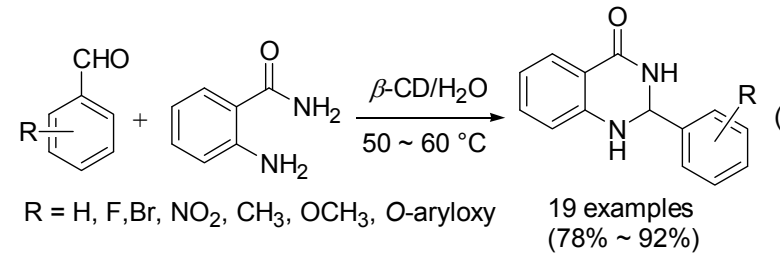

Kumar 等 ${ }^{[11]}$ 将环糊精作为超分子催化剂催化色胺 酮的合成的水相反应(Eq. 3). 他们首先尝试在甲醇、氯 仿、DMF、DMSO、水等溶剂中加入环糊精催化该反应, 发现在水作为溶剂时的收率最高. 继续比较了 $\alpha, \beta$-和 $\gamma-\mathrm{CD}$ 三种环糊精, 只有在使用 $\beta$ - $\mathrm{CD}$ 作为催化剂时反应 的产率最好. 铜催化的叠氮化物与炔烃的 1,3 环加成反 应是一个经典的 Click 反应, Lim 等 ${ }^{[12]}$ 添加催化剂量的环 糊精( $2.5 \mathrm{~mol} \%$ ), 在水相中高效地进行了 Click 反应, 反 应时间大大缩短至分钟级别, Kumar 还继续尝试了直接 使用溴代物作为反应底物，加入叠氮化钠、炔烃化合物 在已摸索的最佳反应条件下一锅法合成三唑衍生物，加 入环糊精也取得很好的收率(Eq. 4). 


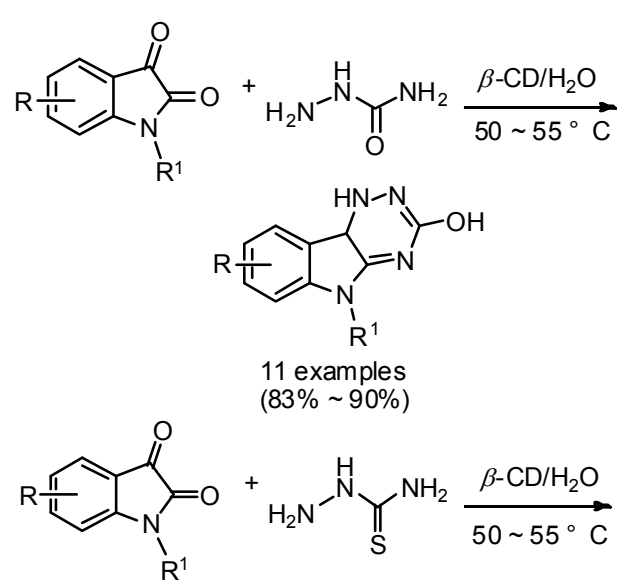

$\mathrm{R}=\mathrm{H}, \mathrm{F}, \mathrm{Br}, \mathrm{Cl}, \mathrm{CH}_{3}, \mathrm{OCH}_{3}, \mathrm{NO}_{2}$

$\mathrm{R}^{1}=\mathrm{H}, \mathrm{CH}_{3}, \mathrm{CH}_{2} \mathrm{C}_{6} \mathrm{H}_{5}$

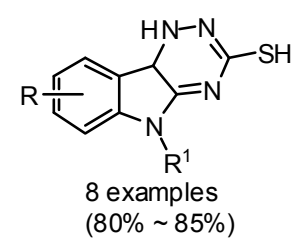

图式 3 环糊精催化吲哚醌与氨基腿/氨基硫腿加成反应

Scheme 3 Isatin and semicarbazide/amino thiourea addition reaction catalyzed by $\mathrm{CD}$

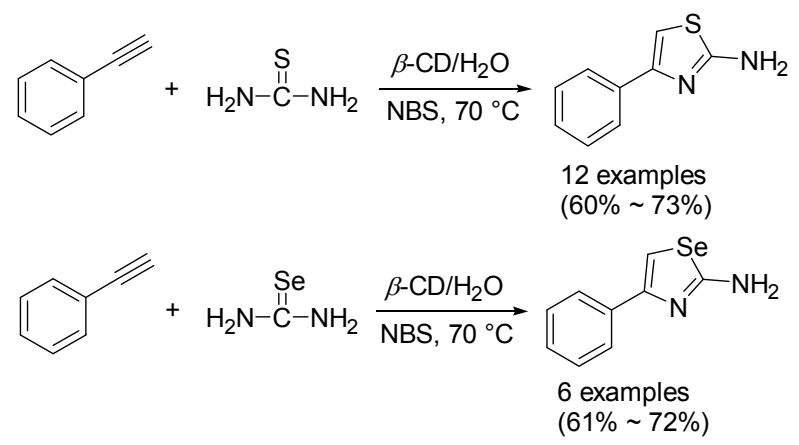

图式 4 环糊精催化合成噻唑、硒唑

Scheme 4 Synthesis of thiazole and selenazole catalyzed by CD<smiles>[R]c1cc2[nH]c(=O)oc(=O)c2cc1[R]</smiles>

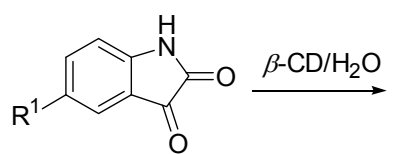<smiles>[R]c1cc2c(cc1[R])C(=O)n1c(nc3cc([R])c([R])cc3c1=O)C2=O</smiles>

23 examples (79\% 90\%)

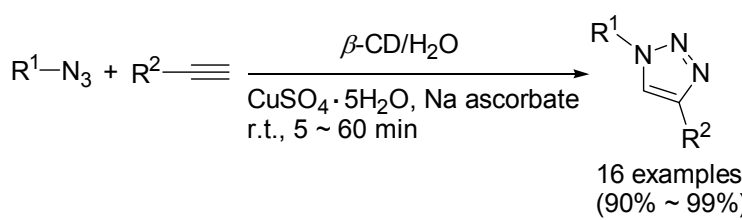

$\mathrm{Ji}$ 等 ${ }^{[13]}$ 利用次氯酸钠作为氧化剂、 $\beta$-环糊精作为催 化剂, 在水中缓和氧化醇成醛. 这个反应无需金属催化 且在水相中就可以进行. 传统的氧化是在水和丙酮的混 合溶剂下使用 2-碘酰基苯甲酸(IBX)作为氧化剂以及在 水溶液中使用 $N$-溴代琥珀酰亚胺(NBS)氧化醇成醛. 他 们这个催化体系对醇氧化不仅有底物选择性、 $\mathrm{NaOCl}$ 比 IBX 价格低廉, 而且反应仅需要在水溶液中就可进行. 结果表明 $\beta$-环糊精在对反应底物的选择上空间因素远 大于电荷性等因素, 其对于苯甲醇生成苯甲醛的产率可 达 $99 \%$ 以上，而对脂肪族醇和 2-金刚烷醇等催化活性大 为降低. $\mathrm{Ji}$ 等还对 $\beta-\mathrm{CD}$ 的超分子催化机理进行初步的探 索, 苯乙醇在该催化体系下反应 $1 \mathrm{~h}$ 后分别加入 $5 \mathrm{~mL}$ 乙 腈和丙酮，可以得到不同的反应结果. 加入乙腈的反应 可以得到 $70 \%$ 的苯乙醛反应产率，而加入丙酮的反应仅 得到 $1 \%$ 的反应产率. 使用丙酮比乙腈对苯乙醇氧化反 应速率有更大的影响，表明在反应过程中，氢键起了很 重要的作用. 由于苯乙醇与 $\mathrm{H}_{2} \mathrm{O}$ 的氢键较弱, 在丙酮和 水的强氢键作用下, 苯乙醇、 $\mathrm{ClO}^{-}$和 $\mathrm{H}_{2} \mathrm{O}$ 的包合物难于 形成(Eq. 5).

$$
\begin{array}{rl}
\mathrm{R} & \mathrm{OH} \underset{\mathrm{NaOCl}, 50{ }^{\circ} \mathrm{C}}{\stackrel{\mathrm{CD} / \mathrm{H}_{2} \mathrm{O}}{\longrightarrow}} \mathrm{R} \\
& \begin{array}{l}
10 \text { examples } \\
(0 \sim 99 \%)
\end{array}
\end{array}
$$

虽然环糊精空腔可以包合疏水有机小分子，催化有 机反应在水相中进行, 但并不是环糊精催化的有机反应 都必须在水溶液反应中. Dalal 等 ${ }^{[14]}$ 用环糊精催化腈类化 合物与叠氮盐环加成生成四唑的水相反应，该反应即使 增加催化剂的量和延长反应时间，反应也几乎没有进 行. 在非水相溶剂中, 如在 DMSO、DMF 等, 有机小分 子与环糊精在超分子相互作用下能够形成分子键得到 超分子溶胶(Eq. 6). 在水相中, 环糊精不能形成超分子 溶胶, 这可能是导致反应不能进行的原因. 而将溶剂换 成 DMF，反应产率却高达 $80 \%$ 以上. 从 DMF- $d_{6}$ 作为溶 剂的氢谱中, 可以明显观察到一些化学位移的变化, 这 是由于环糊精的络合作用引起的. Fernandes 等 ${ }^{[15]}$ 使用 $\beta$-CD 环糊精作为固相催化剂催化 Biginelli 反应实现无 溶剂合成, 此反应无需溶剂, 不含金属且能够得到很高 的产率. 作者尝试了甲醇、乙腈、DMSO、 $\mathrm{H}_{2} \mathrm{O}$ 等溶剂 在其对应的沸点下反应, 得到的收率均比无溶剂、 $100{ }^{\circ} \mathrm{C}$ 条件下低. 作者还对比了 $\alpha-, \beta-, \gamma-\mathrm{CD}, \mathrm{HP}-\gamma-\mathrm{CD}$ 和 Me- $\gamma$-CD 五种环糊精及其衍生物在无溶剂条件下对 反应的影响, 实验结果表明只有在使用 $\beta$-CD 作为催化 剂时, 反应的收率最高(Eq. 7). 


$$
\mathrm{NaN}_{3}+\mathrm{R}-\mathrm{C} \equiv \mathrm{N} \frac{\beta-\mathrm{CD}}{\mathrm{NH}_{4} \mathrm{Cl}, \mathrm{DMF}, 120^{\circ} \mathrm{C}} \underset{\mathrm{R}^{2}}{\stackrel{(76 \% \sim 95 \%)}{\text { examples }}}
$$

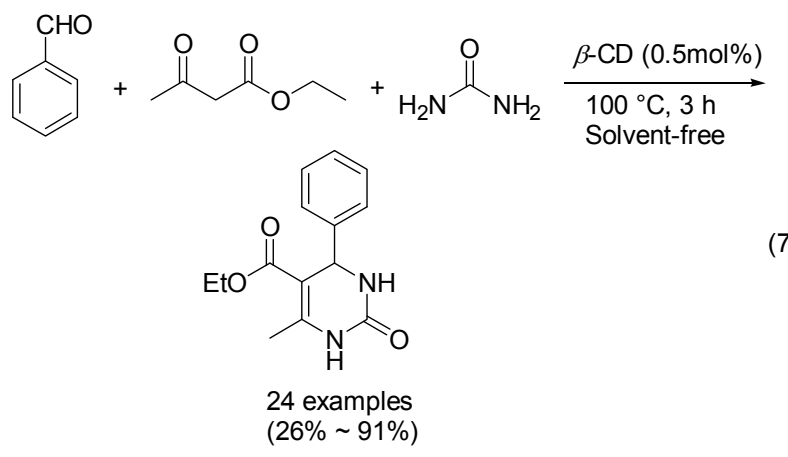

\section{2 金属离子参与的环糊精催化有机反应}

过渡金属催化有机反应是现代有机合成中重要的 手段之一, 将金属离子引入环糊精体系可以有效地结合 金属的催化性能和环糊精的分子识别和相转移等性能. 环糊精空腔主客体以及其生物酶的特性能够催化一系 列的反应，“内疏水、外亲水” 的特性能实现有机反应 在水相中进行. 在环糊精上修饰上 $\mathrm{N} 、 \mathrm{P}$ 原子以及其他 具有孤对电子对、易于与金属配位的原子可使环糊精作 为配体，与金属形成络合物作为催化体系参与催化反 应.

在酶催化的模拟中, Breslow 等 ${ }^{[16]}$ 通过钴-碳键将钴 胺素键合到 $\beta$ - $\mathrm{CD}$ 的伯羟基上, 准确地模拟了天然酶-维 生素 B12 的部分功能进行自由基机理的催化重排反应. 钴胺素修饰的环糊精与腺苷钴胺素的成键较为相似, 这 归因于环糊精的拥挤的空腔系统. 钴胺素修饰的环糊精 能够快速地分解为维生素 B12 与环糊精自由基, 维生素 B12 与环糊精自由基也能化合成钴胺素修饰的环糊精, 这是一个平衡的可逆反应. 这个平衡实际上是一个具有 高活性的辅酶-酶复合物的模型, 它比单纯的腺苷钴胺 素有更高的活性. Karakhanov 等 ${ }^{[17]}$ 对环糊精纳米反应器 进行了另一种改造, 这种连有钯金属离子的环糊精用于 催化 Wacker 氧化反应, 可以使长链的端基烯烃选择性 地氧化成对应的甲基酮. 他们反应条件是在高压灭菌的 钢瓶中, 在 $30 \sim 90{ }^{\circ} \mathrm{C}$ 水溶液下反应时间 $2 \mathrm{~h}$, 反应保持 $0.5 \mathrm{MPa}$ 的氧气压力. 反应除了加入环糊精衍生物作为 配体、钯金属离子作为催化剂, 还加入 $\mathrm{CuCl}_{2}$ 作为辅催 化剂, 这样一个催化体系能够催化不饱和烯烃的选择性 氧化. 催化实验结果表明, 超分子催化剂的活性取决于 超分子空腔与底物分子的契合度, 选择新型超分子以及 对其修饰成大环受体是未来具有选择性超分子催化剂
的研究热门.

Monflier 等 ${ }^{[18]}$ 在甲基化 $\beta$ - $\mathrm{CD}$ (RaMe- $\beta$-CD) 催化有 机反应方面做了大量详细的工作. Monflier 通过合成甲 基化的环糊精，利用它各种优异的特性在各种过渡金属 离子条件下催化有机反应，成功完成了在过渡金属 $\mathrm{Pd}$ 参与下 $\mathrm{RaMe}-\beta-\mathrm{CD}$ 催化 Heck 反应 ${ }^{[18 b]}$ 、 Suzuki- miyama 反应等 ${ }^{[19]}$. 他们选择的 Heck 反应是丙烯酸二乙酯的芳 基化反应，底物采用丙烯酸二乙酯以及活性较大的碘 苯. 反应篮选的最佳条件是在催化剂为 $0.5 \mathrm{~mol} \%$ $\left[\mathrm{Pd}\left(\mathrm{NH}_{3}\right)_{4} \mathrm{Cl}_{2}\right]$ 、碱为 1.5 equiv. 的二异丙基胺以及一定摩 尔量的 RaMe- $\beta$-CD 在水相中 $90{ }^{\circ} \mathrm{C}$ 反应 $6 \sim 8 \mathrm{~h}$, 反应不 仅得到很好的转化率, 产物也具有选择性, 并且底物的 基团耐受性较好. Monflier 合成了第一例环糊精修饰二 齿氮杂环卡宾配体 (bis-NHC) 和二齿介离子卡宾配体 (bis-MIC), 环糊精上剩余羟基分别用甲基或苄基保护. 通过这四种环糊精衍生物作为配体与 $\mathrm{Pd}^{\mathrm{II}}$ 形成配合物, 将此催化体系催化 Suzuki-miyama 碳碳偶联反应, 取得 很好的收率; 在过渡金属纳米 $\mathrm{Ru}$ 催化糠醛催化加氢反 应中, $\mathrm{RaMe}-\beta-\mathrm{CD}$ 可以极大增加纳米 $\mathrm{Ru}$ 的催化效果 ${ }^{[20]}$. 在反应中只加入聚吡乙酰(PVP)作为催化剂 $\mathrm{Ru}$ 的稳定 剂时, 反应的转化率只有 $30 \%$. 当 PVP 与 $\mathrm{RaMe}-\beta-\mathrm{CD}$ 以 $8: 2$ 的物质的量比加入反应，反应的转化率达到 $53 \%$, 若加入的是 RaMe- $\gamma-\mathrm{CD}$ 可提高至 $61 \%$. 由实验数 据可以看出, 合成的 $\mathrm{RaMe}-\mathrm{CD}$ 可增加纳米 $\mathrm{Ru}$ 催化糠醛 催化加氢生成糠醇的反应.

他们还通过 Click 反应在环糊精修饰上 P 配体且膦 原子上带有两个位阻较大的苯环, 将合成的的环糊精 $\mathrm{P}$ 配体在金属 $\mathrm{Rh}$ 参与下催化加氢甲酰化反应. 再通过加 入金刚烷客体分子可以影响反应的方向，得到两种不同 的主要产物 ${ }^{[21]}$. 在未加入金刚烷客体分子时，反应生成 的主要产物是直链醛类，当加入金刚烷客体分子，反应 生成的主要产物是支链醛类. 这个配体的构象是可控制 的，在加氢甲酰化反应中通过加入客体分子金刚烷酸钠 可以导致产物区域选择性的变化(Scheme 5).

Pitchumani 等 ${ }^{[22]}$ 合成了环糊精 $\mathrm{C}(6)$ 上伯羟基全修饰 上氨基的 $\beta$ - $\mathrm{CD}$ (Per-6- $\mathrm{ABCD}$ ), 在金属 $\mathrm{Cu}$ 的催化下，可 以高效的催化许多有机反应. Per-6-ABCD 作为配体和 主体在 $\mathrm{CuI}$ 作为催化剂高效催化咪唑与芳基澳代物的反 应(Eq. 8). Pitchumani 猜测反应机理可能是修饰的环糊 精先与金属离子 $\mathrm{Cu}^{+}$形成络合物, 然后芳基溴代物进入 环糊精的疏水空腔发生氧化加成与金属铜形成络合物, 接着咪唑进行配体交换并发生还原消除得到目标产物. Per-6-ABCD/CuI 还可以催化卤代芳烃发生氧化反应生 成芳烃氰化物 ${ }^{[23]}$. 此反应的反应机理与其研究环糊精催 化有机反应的机理大致相同，不同点在于此反应的芳基 


$$
\mathrm{R} \curvearrowright \frac{\mathrm{Rh} / \text { Ligand } 1}{\mathrm{CO} / \mathrm{H}_{2}(V: V=1: 1)} \mathrm{R} \frown \mathrm{CHO} \text { or }
$$<smiles>[R][R]OC(=O)CCC</smiles>

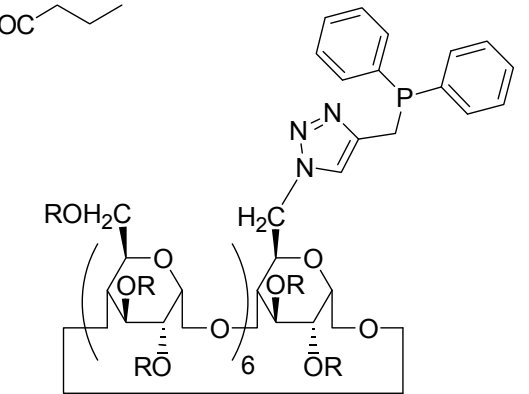

$\mathrm{R}=\mathrm{H}$ or $\mathrm{CH}_{3}$ (DS: 1.8 per glucose unit)

Ligand 1

图式 5 环糊精衍生物作为配体参与 $\mathrm{Rh}$ 催化的加氢甲酰化反 应

Scheme 5 Hydroformylation reaction catalyzed by $\mathrm{Rh}$ and cyclodextrin derivatives as ligand

卤化物与金属络合时是发生在环糊精的空腔外, 证据是 这个反应在水以及低极性溶剂如甲苯和乙腈作为溶剂 下几乎不发生反应, 在溶剂 DMF 中才达到了高的产率 (Eq. 9).

$$
\begin{aligned}
& \underset{\mathrm{H}}{\mathrm{Br}}+\underset{\mathrm{K}_{2} \mathrm{CO}_{3}, \mathrm{DMSO}, 110^{\circ} \mathrm{C}}{\mathrm{N}} \\
& \text { (81\% 98\%) } \\
& \overbrace{\substack{\mathrm{K}_{4}\left[\mathrm{Fe}(\mathrm{CN})_{6}\right] \\
\mathrm{Na}_{2} \mathrm{CO}_{3}, \mathrm{DMF}, 130^{\circ} \mathrm{C}}}^{\mathrm{Cul} / \mathrm{Per}-\mathrm{ABCD}} \underset{\begin{array}{l}
21 \text { examples } \\
(70 \% \sim 97 \%)
\end{array}}{\mathrm{CN}}
\end{aligned}
$$

Kaboudin 等 ${ }^{[24]}$ 合成二价铜离子与环糊精的包合物 $\left(\mathrm{Cu}_{2}-\beta-\mathrm{CD}\right)$, 该包合物不是简单的物理混合, $\mathrm{Cu}$ 与环糊 精上的羟基是键合在一起. $\mathrm{Cu}_{2}-\beta-\mathrm{CD}$ 可作为纳米催化剂 催化嗍酸酯发生自偶联(Eq. 10)以及苯硣酸、叠氮化钠与
苯乙炔一锅法合成三唑化合物(Eq. 11). Kaboudin 在原来 实验的基础上将纳米催化剂 $\mathrm{Cu}_{2}-\beta-\mathrm{CD}$ 与磁性 $\mathrm{Fe}_{3} \mathrm{O}_{4}$ 结合 得到环糊精与磁性铁的络合物 $\left(\mathrm{Fe}_{3} \mathrm{O}_{4}-\mathrm{Cu}_{2}-\beta-\mathrm{CD}\right)$, 将 $\mathrm{Fe}_{3} \mathrm{O}_{4}-\mathrm{Cu}_{2}-\beta-\mathrm{CD}$ 用于催化硼酸酯发生自偶联反应, 得到 的收率依旧很好, 并且催化剂还能通过强磁铁回收再利 用. $\mathrm{Fe}_{3} \mathrm{O}_{4}-\mathrm{Cu}_{2}-\beta-\mathrm{CD}$ 作为催化剂催化苯硽酸、叠氮化钠 与苯乙炔一锅法合成三唑化合物也同样取得很好的收 率，通过强磁铁将纳米催化剂回收再重新用于反应上， 催化剂重复参与反应 4 次, 反应收率几乎不减少 (Eq. 12).

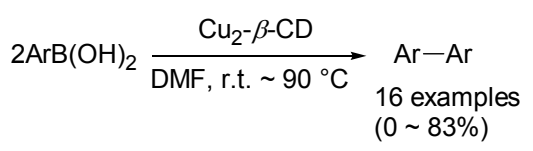

$$
\operatorname{ArB}(\mathrm{OH})_{2} \underset{\text { (2) } \mathrm{R}-\mathrm{C} \equiv \mathrm{CH} \text {, r.t., } 4 \mathrm{~h}}{\stackrel{(1) \mathrm{NaN}_{3}, \mathrm{H}_{2} \mathrm{O}}{\mathrm{Cu}_{2}-\beta-\mathrm{CD} \text {, r.t., } 2 \sim 6 \mathrm{~h}}} \stackrel{\begin{array}{l}
17 \text { examples } \\
(89 \% \sim 97 \%)
\end{array}}{\stackrel{\mathrm{N}=\mathrm{N}}{\mathrm{N}}-\mathrm{Ar}}
$$

$$
\begin{aligned}
& \text { (1) } \mathrm{NaN}_{3}, \mathrm{H}_{2} \mathrm{O} \text {, r.t., } 2 \sim 6 \mathrm{~h} \\
& \mathrm{ArB}(\mathrm{OH})_{2} \frac{\mathrm{Fe}_{3} \mathrm{O}_{4}-\mathrm{Cu}_{2}-\beta-\mathrm{CD}}{(2) \mathrm{R}-\mathrm{C}=\mathrm{CH} \text {, r.t., } 4 \mathrm{~h}} \\
& 21 \text { examples } \\
& \text { (70\% 97\%) }
\end{aligned}
$$

Matt 等 ${ }^{[25]}$ 将环糊精衍生物与贵金属如钯、铑、金等 反应制得金属离子与环糊精衍生物的螯合物，用于催化 有机反应，能够取得很好的收率。他们在环糊精 C-2、 C-3 上修饰一个手性的磷酸盐, 该含 $\mathrm{P}$ 的环糊精衍生物 与 $\left[\mathrm{Rh}(\mathrm{acac})(\mathrm{CO})_{2}\right](\mathrm{acac}=$ acetylacetonate $)$ 在溶剂 $\mathrm{CH}_{2} \mathrm{Cl}_{2}$ 中反应，可制得环糊精衍生物与 $\mathrm{Rh}$ 的络合物. 他们制 备了 $\alpha-, \beta-\mathrm{CD}$ 两种催化体系, 其中 $\beta-\mathrm{CD}$ 催化体系制备 如 Eq. 13, 并运用于 $\mathrm{Rh}$ 催化苯乙烯加氢甲酰化反应, 能 达到很好的立体选择性, 两个催化体系反应 ee 值可高 达 95\%、92\% ${ }^{[25 \mathrm{a}]}$.

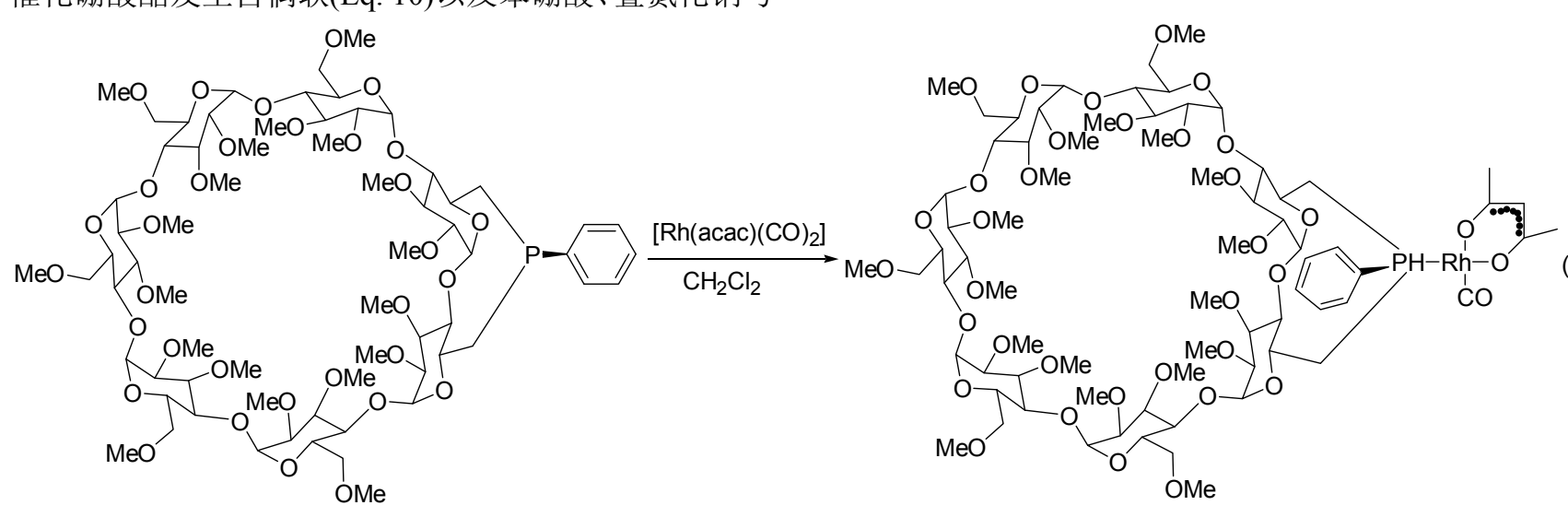


在环糊精配体催化方面, 我们 ${ }^{[26]}$ 也报道了以手性氨 基酸修饰的环糊精作为配体催化的水相 Heck 反应(Eq. 14). 三种氨基酸通过氨基和环糊精相链接, 形成了氨基 酸修饰的环糊精配体. 通过条件优化, 在醋酸钯的催化 下，实现了水相条件下的 Heck 反应，产率高达 $97 \%$. 并 且该催化体系可以实现多次回收利用, 有效地降低催化 成本, 具有一定的工业应用价值. 同时大部分氨基酸是 手性化合物, 可以作为手性配体进行催化反应, 为手性 合成提供了可能，目前该工作我们正在进行中.
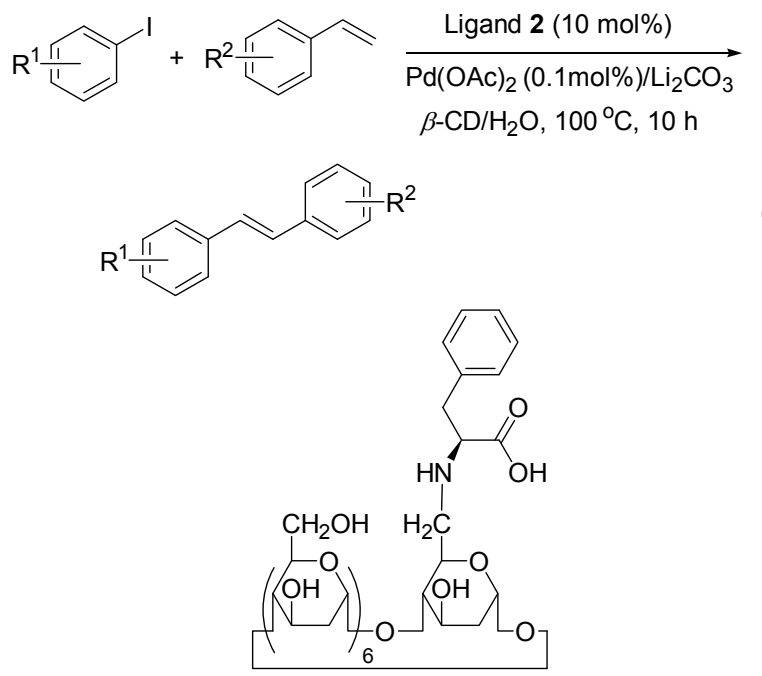

Ligand 2

\section{3 无金属离子参与的环糊精催化有机反应}

过渡金属催化有机反应虽然能取得高产率，但是多 数金属都价格昂贵, 并且具有毒性, 不符合绿色化学的 要求. 环糊精及其衍生物也能在无金属参与的条件下催 化部分水相反应.

Bols 等 ${ }^{[27]}$ 在环糊精上修饰了腈醇基、乙烯基、乙酸 基等制备得到一系列的环糊精衍生物, 他们选择修饰的 官能团是与人体内一些活性酶类似, 得到的环糊精衍生 物具有类人工酶的特性, 能够选择性地催化一部分反 应. Bols 在 $\beta$-环糊精的 2 位着基上修饰 2 4 个甲酰甲基 (Scheme 6) 制得三类环糊精衍生物 $\mathbf{3} \sim \mathbf{6}$, 将这三类环糊 精在中性的缓冲溶液下催化氧化氨基酚的反应, 并以过 氧化氢作为氧化剂, 2 -氨基苯酚作为底物时, 反应收率 增加了 20000 倍. 当他们将邻氨基甲酚和对氨基甲酚一 起加入反应, 且反应时间足够长时, 两个底物的反应收 率比为 $16: 1$. 说明制备的衍生物是具有很好的底物选 择性的, 类人工酶选择性氧化研究有很好的前景.

$\mathrm{Ji}$ 等 ${ }^{[28]}$ 就无金属离子参与的环糊精催化有机反应做 了很多的研究. 他们将 2 位修饰羟丙基的 $\beta$-环糊精 (2-HP- $\beta-\mathrm{CD})$ 与纤维素修饰在一起做成聚合物(2-HP- $\beta$ -

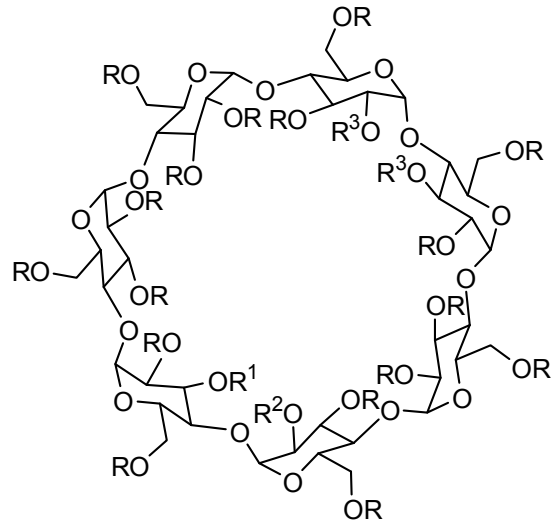

$$
\begin{aligned}
& 3 \mathrm{R}=\mathrm{H}, \mathrm{R}^{1}=\mathrm{CH}_{2} \mathrm{CHO}, \mathrm{R}^{2}=\mathrm{R}^{3}=\mathrm{H} \\
& 4 \mathrm{R}=\mathrm{Me}, \mathrm{R}^{1}=\mathrm{CH}_{2} \mathrm{CHO}, \mathrm{R}^{2}=\mathrm{R}^{3}=\mathrm{Me} \\
& 5 \mathrm{R}=\mathrm{Me}, \mathrm{R}^{1}=\mathrm{R}^{2}=\mathrm{CH}_{2} \mathrm{CHO}, \mathrm{R}^{3}=\mathrm{Me} \\
& 6 \mathrm{R}=\mathrm{Me}, \mathrm{R}^{1}=\mathrm{R}^{2}=\mathrm{R}^{3}=\mathrm{CH}_{2} \mathrm{CHO}
\end{aligned}
$$

图式 6 甲酰甲基修饰的环糊精衍生物

Scheme 6 Cyclodextrin derivatives modified formyl methyl

$\mathrm{CDP}$ ), 该聚合物能够作为生物模拟酶在水相中催化合 成苯甲醛(Eq. 15). 2-HP- $\beta$-CD 通过环氧氯丙烷键合固定 在纤维素上, 由于环糊精和纤维素都是由葡萄糖单元组 成, $2-H P-\beta-C D P$ 具有很好的生物性, 能够作为高效的生 物催化剂催化肉桂醛氧化生成苯甲醛. 虽然聚合物与反 应物间的相互作用较弱，但是该相互作用对反应的影响 依然很大, 在未加入 $2-\mathrm{HP}-\beta-\mathrm{CDP}$ 时, 反应的转化率仅 有 $38 \%$, 在篮选的最佳条件下加入 $2-\mathrm{HP}-\beta$-CDP, 反应转 化率可高达 $98 \%$. 而且反应结束后，催化剂可以无损回 收再利用.

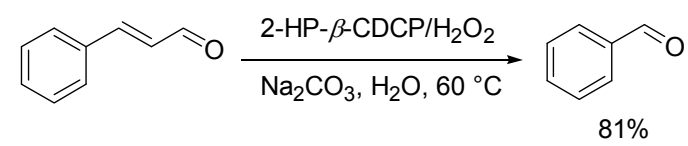

Pitchumani 等 ${ }^{[29]}$ 合成的 Per-6-ABCD 除了在金属参 与下可以高效地催化有机反应，在无金属参与的部分反 应中也能获得很好的收率. Per-6-ABCD 含有的 7 个氨基 使得其具有优良的性质，活泼的氨基易作为亲核试剂进 攻碳原子, 通过电荷性作用影响反应物的反应活性. 他 们用 Per-6-ABCD 催化合成吡喃衍生物的无溶剂反应, 反应只需研磨 1 7 min 就可以达到很高的产率(Eq. 16). 反应过程中苯甲醛与丙二腈进入环糊精空腔在环糊精 上氨基的作用下发生 Knoevenagel 缩合反应, 接着环己 二酮进入环糊精空腔发生 Machael 加成反应，最后发生 环加成反应生成最终产物. 在多组分反应合成吡唑类化 合物的反应中, 反应在有机溶剂 DMF、DMSO 反应 $24 \mathrm{~h}$, 反应产物的产率仅有 $58.2 \%$ 、 $61.6 \%$. 而在无溶剂、 Per-6-ABCD 作为催化剂的条件下, 反应只需研磨 $1 \mathrm{~min}$ 即可获得高达 $99 \%$ 的产率 ${ }^{[30]}$ (Eq. 17). 


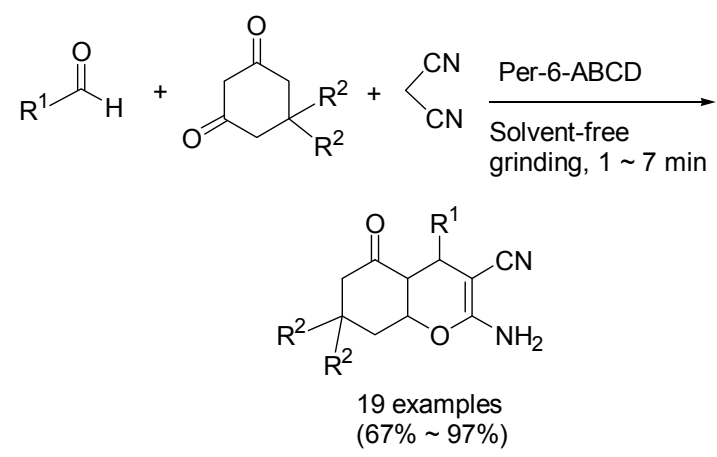

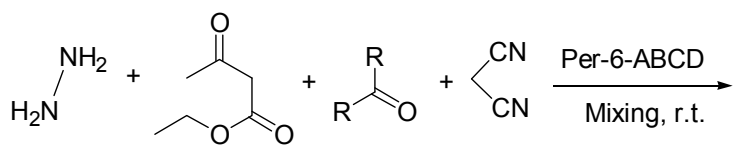<smiles>[R]C12[W]OC1([R])c1c(n[nH]c1C)OC(N)=C2CN</smiles>

\section{4 环糊精衍生物应用于手性催化反应}

环糊精是由糖单元构成, 是天然的手性主体分子. 环糊精上的羟基具有手性，对环糊精进行化学修饰，可 制得具有手性的环糊精衍生物. 这样的衍生物在不对称 催化反应领域有一定的应用, 也能够作为手性配体参与 过渡金属手性催化有机反应. 不对称催化技术在医药、 工业、环境、新材料等领域有着重要的作用, 这使得环 糊精在不对称催化上有着重要的研究价值.

将不对称化合物引入到催化剂体系中是一种普遍 使用的引入手性中心的方法. Cheng 等 ${ }^{[31]}$ 报道了环糊精 催化的不对称合成反应, 手性邻二胺修饰的环糊精作为 催化剂, 成功地催化了手性 Aldol 反应, 其 $e e$ 值最高达 到 $97 \%$. 他们合成了 5 种手性邻二胺修饰的环糊精和乙 二胺修饰的环糊精, 将这 6 种环糊精衍生物和 4 个带有 烷基链的手性邻二胺通过实验篮选出最佳配体 7, 其反 应产物的 $e e$ 值高达 $97 \%$. 将不对称超分子伯胺催化剂通 过共价键嫁接到环糊精主体上被证明是一个不对称超 分子催化剂的发展方向(Scheme 7).

$\mathrm{Ji}$ 等 ${ }^{[32]}$ 将环糊精进行手性醇胺修饰, 和 $\mathrm{Ru}$ 形成配 合物作为催化剂, 催化芳香酮成醇的手性反应, 产率和 $e e$ 值都达到了 $80 \%$. Ji 认为底物在环糊精空腔内反应时 有下面两种模型——模型 $\mathrm{A}$ 和模型 $\mathrm{B}$ (图 2). 人工酶以及 基于环糊精的人工酶应用于不对称有机反应往往达不 到满意的结果. 他们将修饰后的环糊精和金属催化剂 $\left[\mathrm{RuCl}_{2} \text { (Benzene) }\right]_{2}$ 和 $\left[\mathrm{RuCl}_{2} \text { (Mesitylene) }\right]_{2}$ 配位, 这样一 个人工酶催化体系应用于手性芳香酮加氢反应取得了
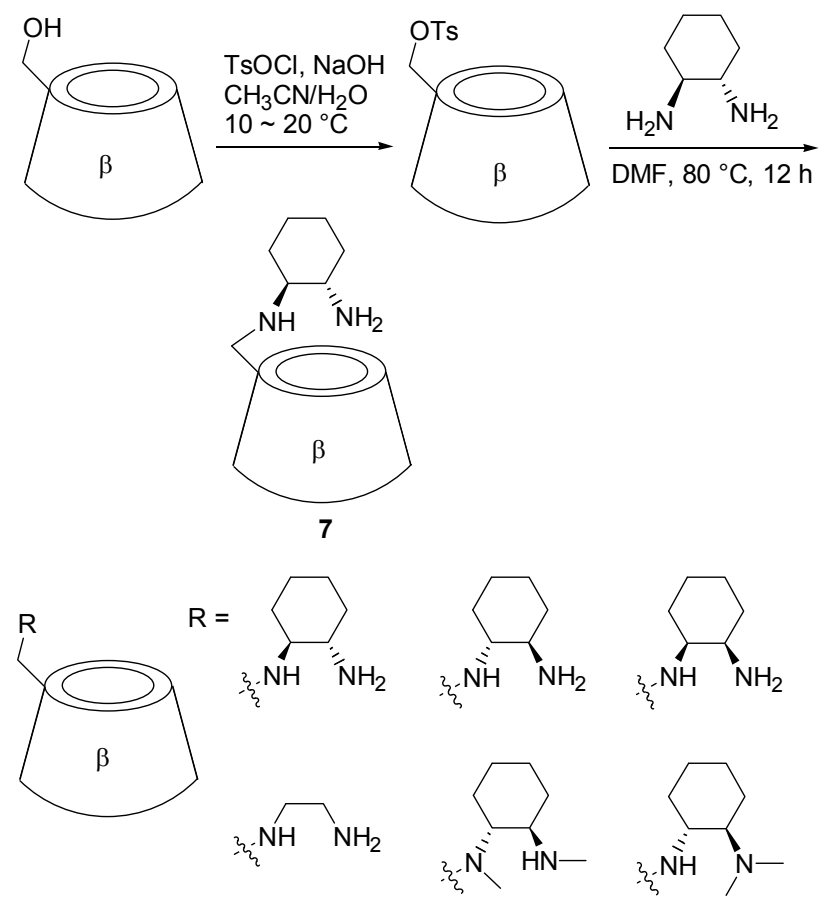

图式 7 手性胺修饰的环糊精衍生物

Scheme 7 Cyclodextrin derivatives modified chiral amine

较好的收率. 而且环糊精与 $\left[\mathrm{RuCl}_{2}(\mathrm{Mesitylene})\right]_{2}$ 配位时 比其与 $\left[\mathrm{RuCl}_{2} \text { (Benzene) }\right]_{2}$ 配位催化反应产物的 ee 值更 高, 这是因为以模型 $\mathrm{B}$ 的方式反应得到产物是外消旋 体,增大金属 $\mathrm{Ru}$ 的芳基配体体积时，阻止了底物以模型 $\mathrm{B}$ 的方式反应.

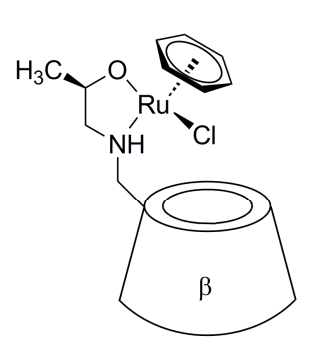

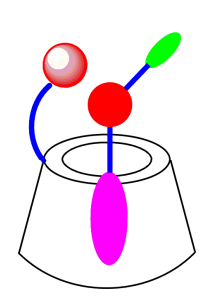

A

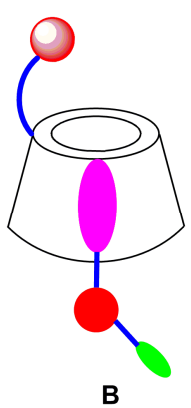

B
图 2 手性醇胺修饰的环糊精衍生物

Figure 2 Cyclodextrin derivatives modified chiral alcohol amine

Sollogoub 等 ${ }^{[33}$ 将氮杂环卡宾修饰在环糊精上, 制 得具有手性的环糊精配体, 然后将金属 $\mathrm{CuCl} 、 \mathrm{Ag}_{2} \mathrm{O}$ 、 $\mathrm{AuCl}$ 分别与环糊精形成络合物, 得到三个具有手性的 催化体系. 将 $\mathrm{AuCl}-\mathrm{CD}$ 催化体系催化烯炔反应生成具 有手性的二环化合物(Eq. 18). 通过 NMR 确定了金属离 子是在环糊精空腔内起催化作用, 尤其是环糊精上 H-5 化学位移发生最大变化, 而 H-3 受到卤素原子配体以及 氮杂卡宾环的屏蔽作用化学位移未发生太大变化. 金属 离子与卤素复合物形成一个独特的键 $\mathrm{C}-\mathrm{H} \cdots \mathrm{M}, \mathrm{C}-$ 
$\mathrm{H} \cdots \mathrm{X}-\mathrm{M}$, 和 $\pi \cdots \mathrm{X}-\mathrm{M}$.
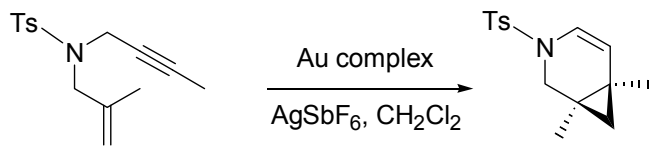

$77 \%, 59 \%$ ee

Pitchumani 等 ${ }^{[34]}$ 在手性催化有机反应上深入的研 究, 合成的 Per-6-ABCD 上的氨基可作为手性源, 能够 催化一部分反应使得产物具有手性且 $e e$ 值很高. 这不 仅是因为环糊精衍生物是手性分子, 还有环糊精空腔能 与有机小分子形成包合物以及环糊精提供极大的位阻 效应等原因. Per-6-ABCD 作为一个手性主体超分子催 化剂能够高效催化 Henry 反应(Eq. 19), 在无需金属参与 的条件下, 产物 ee 值达到 $98 \%$ 以上, 并且催化剂能够无 损回收重复再利用. Per-6-ABCD 还能催化不对称合成 喹啉类化合物 ${ }^{[35]}$ (Eq. 20). 邻氨基苯乙酮与醛在乙醇和 水的混合溶剂下，温度控制在 $-5{ }^{\circ} \mathrm{C}$ 反应 $15 \mathrm{~h}$, 反应产 物是具有手性的喹啉类化合物且收率和 $e e$ 值最高达 99\%, 99\%.

$$
\begin{aligned}
& \text { Per-6-ABCD } \\
& \begin{array}{l}
\mathrm{ACN} / \mathrm{H}_{2} \mathrm{O} \\
7 \mathrm{~h},-2{ }^{\circ} \mathrm{C}
\end{array}
\end{aligned}
$$

\section{5 环糊精及其衍生物应用于光化学反应}

环糊精是由葡萄糖单元组成的, 是天然的手性主体 分子，而且没有芳基等吸收基团，基本上是紫外透明的. $\alpha-, \beta$-和 $\gamma-\mathrm{CD}$ 的空腔能够提供不同大小的空腔, 可以包
结不同大小的客体分子并且环糊精价格低廉、无毒易得, 这些优点使得环糊精及其衍生物成为光化学反应研究 的热门, 且环糊精也是最早应用于不对称光化学反应的 手性主体之一 ${ }^{[36]}$.

Brinker 等 ${ }^{[37}$ 研究了叠氮金刚烷在 $\alpha-, \beta-\mathrm{CD}$ 以及无 $\mathrm{CD}$ 条件下的光分解反应. 叠氮金刚烷在环糊精空腔内 反应会得到不同的反应结果，这可以深入探究超分子封 装对反应的影响. 叠氮金刚烷在无 $\mathrm{CD}$ 存在条件下只生 成产物 $\mathbf{A}$, 在 $\alpha-\beta-\mathrm{CD}$ 存在的条件下得到主要产物是化 合物 $\mathbf{A}$, 产率分别为 94\%、91\%。通过 2D-ROESY 看出 叠氮金刚烷与环糊精能够较好地包合，证明了叠氮金刚 烷是在环糊精空腔的影响下，得到不同的反应结果 (Scheme 8).

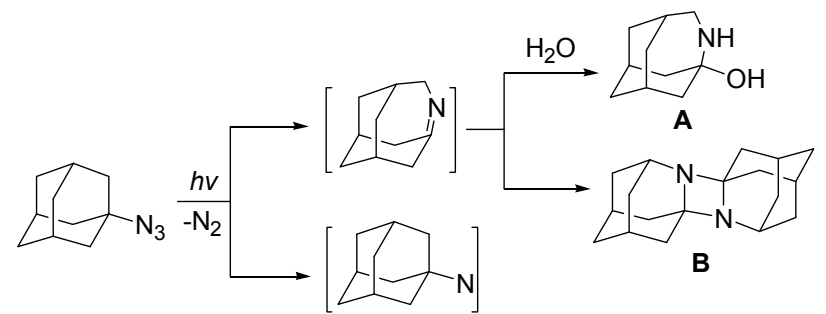

图式 8 叠氮金刚烷的光分解反应

Scheme 8 Photolysis reaction of azide adamantane

Monti 等 ${ }^{[38]}$ 研究了酮洛芬(KP)在环糊精空腔里反应 光脱羧反应. 他们通过圆二色谱、等温滴定量热法、核 磁共振等研究环糊精对 $S$-、 $R$-酮洛芬的手性识别能力. 结合分子热力学、动力学以及量子力学分析 $\mathrm{KP}: \beta-\mathrm{CD}$ 的包合结构(Scheme 9).

Ikeda 等 ${ }^{[39]}$ 对环糊精催化的光促进不对称反应进行 研究. 他们在 $\gamma-\mathrm{CD}$ 上修饰两个吡啶基团，作为超分子反 应器控制 2-蒽甲酸光促环化二聚反应的立体选择性. 当 A,E-2-吡啶- $\gamma$-CD 加入 2-蒽甲酸的光促环化二聚反应中, 头对头的反式异构体聚合产物产率比没有加环糊精或 者加入未修饰的 $\gamma$-CD 的产率要高(Scheme 10).

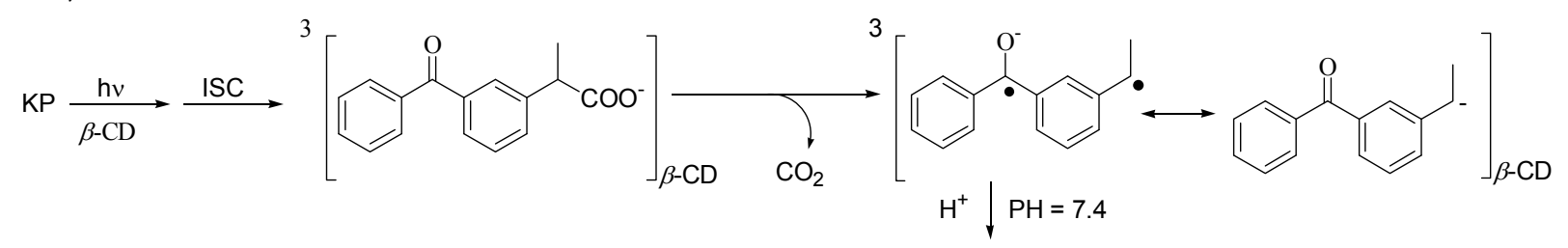

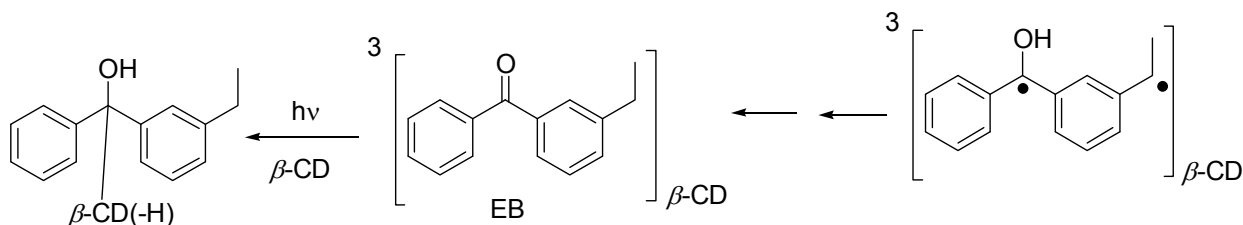

图式 9 酮洛芬在环糊精空腔里反应光脱羧反应

Scheme 9 Decarboxylation reaction of KP in the CD cavity 


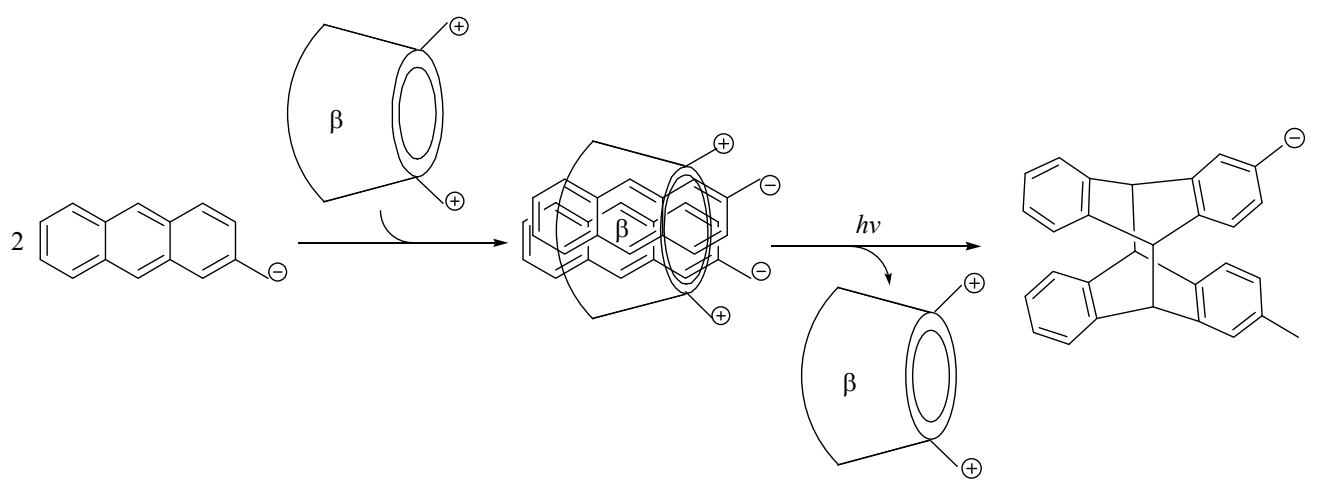

图式 10 环糊精催化不对称的光催化反应

Scheme 10 Photocatalytic asymmetric reaction catalyzed by CD

Inoue 等 ${ }^{[40]}$ 进一步对环糊精催化的光促进不对称反 应进行了系统地研究, 对于 2-葱甲酸在水相介质中进行 立体结构可控的光促环化二聚反应, 产率 $98 \%, e e$ 值达 到 $99 \%{ }^{[41]}$. 他们在 $\alpha-\mathrm{CD}$ 上修饰两个 2-葱甲酸, 这样双 修饰的 $\alpha-\mathrm{CD}$ 有三种, 分别是 A,B-2-(2-葱甲酸) $\alpha-\mathrm{CD}$ (8)、 $\mathrm{A}, \mathrm{C}-2-(2-$ 葱甲酸) $-\alpha-\mathrm{CD}$ (9) 和 A,D-2-(2- 萝甲 酸)- $\alpha-\mathrm{CD}(\mathbf{1 0})$. 两个 2 -葱甲酸发生光促环化二聚反应可 生成 4 种同分异构体 11、12、13、14. 其中 12、13 是 具有手性的. 在水相反应中加入 $\gamma-\mathrm{CD}$, 对三种 $\alpha-\mathrm{CD}$ 环 糊精衍生物上 2 - 葱甲酸的环化二聚反应产率以及 $e e$ 值 都有很大的影响. $\gamma-\mathrm{CD}$ 的大环空腔能够包裹两个 2 -葱甲 酸, 反应受到空腔氢键影响产生不同的反应结果. 反应
物 $\mathbf{8}$ 在加入 $\gamma-\mathrm{CD}$ 后, 生成物 $\mathbf{1 3}$ 的产率只有 $23 \%, e e$ 值 只有 $64 \%$. 反应物 9 在加入 $\gamma-\mathrm{CD}$ 后, 生成物 $\mathbf{1 3}$ 的产率 只有 $98 \%$, ee 值达 $99 \%$. 而反应物 $\mathbf{1 0}$ 加入 $\gamma-\mathrm{CD}$ 后, 生 成物 13 的产率只有 $11 \%, e e$ 值只有 $60 \%$ (Scheme 11).

\section{6 总结与展望}

综上所述, 环糊精及其衍生物能够将有机小分子等 包合进疏水空腔, 改变有机小分子在水中的溶解性, 还 能通过电荷作用影响分子的电性环境, 使得反应能够在 温和的条件下进行, 也能实现有机反应在水相中进行.

虽然未修饰的环糊精也能提高有机分子的水溶性,<smiles>O=C(O)c1ccc2c(c1)C1c3ccccc3C(c3ccccc3)(c3ccccc3C34c5cc(C(=O)O)ccc5C3c3ccccc3C43c4ccccc4C4(c5ccccc54)c4cc(C(=O)O)ccc43)c3ccccc3C21</smiles>

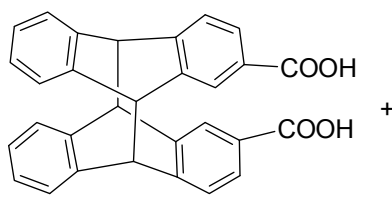

13<smiles>O=C(O)c1ccc2c(c1)C1(c3ccccc3)c3ccccc3C2c2ccccc21</smiles>

15

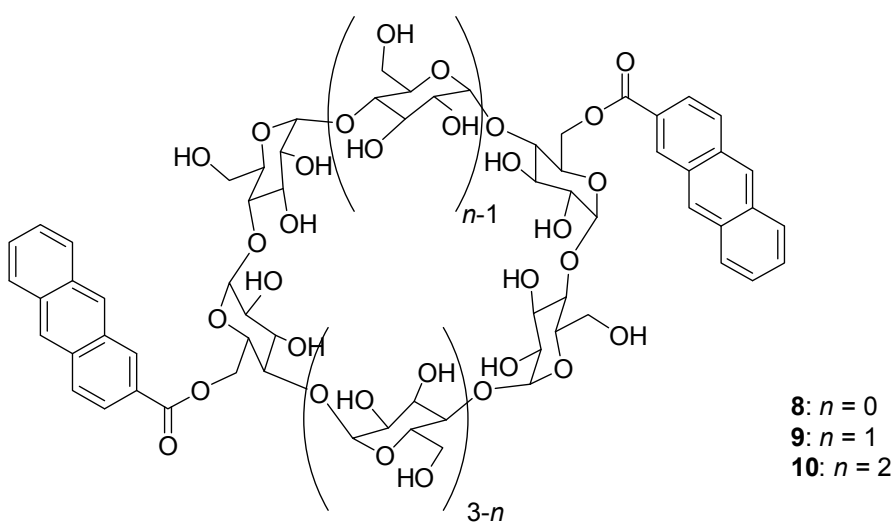

图式 11 环糊精催化二聚反应

Scheme 11 Photocatalytic dimerization reaction catalyzed by CD 
通过主客体作用能够将反应物包合进入空腔催化反应 的进行, 但由于未修饰的环糊精在有机溶剂中溶解性差 以及有限的疏水区域和催化活性, 使得环糊精在应用上 受到局限, 所以需要对环糊精进行化学修饰以弥补环糊 精的不足, 通过对环糊精的修饰, 可以增加环糊精主体 官能团种类和数量, 提高了环糊精催化有机合成反应的 催化活性, 拓展了环糊精催化有机合成反应的应用范 围. 环糊精及其衍生物催化有机反应有时不仅是提供一 个空腔促进反应的加速进行, 而且环糊精上修饰的官能 团能够起到催化效果. 除此之外, 环糊精衍生物还能作 为金属配体，与过渡金属组成催化体系催化有机反应.

环糊精是天然的手性分子, 将环糊精及其衍生物用 于催化有机反应，不仅能使反应在温和的条件下获得良 好的收率，还能运用于不对称合成、手性光化学反应. 而且由于环糊精空腔的影响, 在产物的区域选择性以及 光学选择性都可达到优良的收率. 由于环糊精价廉易 得、无毒具有生物相容性, 而且作为催化剂往往能收回 无损再利用, 所有环糊精是一类性能优良的绿色有机催 化剂. 随着环糊精及其衍生物的催化反应机理研究, 环 糊精在有机合成上的运用会越来越广泛.

\section{References}

[1] (a) Yebeutchou, R. M.; Dalcanale, E. J. Am. Chem. Soc. 2009, 131, 2452 .

(b) Liu, L.; Guo, Q.-X. J. Inclusion Phenom. Macrocyclic Chem. 2002, 42, 1 .

(c) Cheng, P.-F.; Jiao, S.-Y.; Xu, K.-X.; Wang, C.-J. Chin. J. Org. Chem. 2013, 33, 280 (in Chinese).

(程鹏飞, 焦书燕, 徐括喜, 王超杰，有机化学, 2013, 33, 280.)

(d) Zong, Q.-S.; Wu, J.-Y. Chin. J. Org. Chem. 2013, 33, 568 (in Chinese).

(宗乾收，吴建一，有机化学, 2013, 33, 568.)

(e) Liu, B.-Y.; Jin, Y.-X.; Yu, S.-S.; Qu, F.-Y.; Tan, G.-H. Chin. J. Org. Chem. 2013, 33, 1950 (in Chinese).

(粱波颖，金英学，于沙沙，曲凤玉，谭广慧，有机化学, 2013, 33, 1950.)

(f) Liu, H.; Huang, Z.-G.; Wen, K.; Jiang, B. Chin. J. Org. Chem. 2014, 34, 316 (in Chinese).

(刘华, 黄祚刚, 文珂, 姜标, 有机化学, 2014, 34, 316.)

[2] (a) Kaliappan, R.; Kaanumalle, L. S.; Ramamurthy, V. Chem. Commun. 2005, 4056

(b) Kaliappan, R.; Ling, Y.; Kaifer, A. E.; Ramamurthy, V. Langmuir 2009, 25, 8982 .

[3] (a) Klöck, C.; Dsouza, R. N.; Nau, W. M. Org. Lett. 2009, 11, 2595. (b) Jon, S. Y.; Ko, Y. H.; Park, S. H.; Kim, H.-J.; Kim, K. Chem. Commun. 2001, 1938.

(c) Yang, C.; Mori, T.; Origane, Y.; Ko, Y. H.; Selvapalam, N.; Kim, K.; Inoue, Y. J. Am. Chem. Soc. 2008, 130, 8574.

(d) Yang, C.; Ke, C.; Liang, W.; Fukuhara, G.; Mori, T.; Liu, Y.; Inoue, Y. J. Am. Chem. Soc. 2011, 133, 13786.

(e) Yi, J.-M.; Xiao, X.; Zhang, Y.-Q.; Xue, S.-F.; Tan, Z.; Zhang, J.-X. Acta Chim. Sinica 2014, 72, 158 (in Chinese).

(易君明, 肖欣, 张云黔, 薛赛凤, 陶朱, 张建新, 化学学报, 2014, 72, 158.)
[4] (a) Yuan, Z.; Chen, J.; Zeng, Y.; Li, Y.-Y.; Han, Y.; Li, Y. Org. Biomol. Chem. 2011, 9, 6256.

(b) Yuan, Z.; Zheng, S.; Zeng, Y.; Chen, J.; Han, Y.; Li, Y.; Li, Y. New J. Chem. 2010, 34, 718.

[5] (a) Han, C.; Xu, Z.; Diao, C.-H.; Chen, X.; Liu, J.; Gou, M.-J.; Fan, J. Acta Chim. Sinica 2013, 71, 439 (in Chinese).

(韩聪, 徐喆, フ春华, 陈金鍑, 刘靖, 郭敏杰, 樊志, 化学学报, 2013, 71, 439.)

(b) Kong, R.; Shi, D.-J.; Liu, R.-J.; Wu, C.; Ni, P.-H.; Chen, M.-Q. Acta Chim. Sinica 2013, 71, 1540 (in Chinese).

(孔芯，施冬健，刘蓉瑾，吴超，倪沛红，陈明清，化学学报， 2013, 71, 1540.)

(c) Yao, J.-B.; Xu, M.-Y.; Yan, Z.-Q.; Wu, W.-H.; Yang, C. Chin. J. Org. Chem. 2014, 34, 26 (in Chinese).

(姚家斌, 徐梦瑜, 严志强, 伍晚花, 杨成, 有机化学, 2014, 34, 26.)

(d) Shen, H.-M.; Wu, H.-K.; Ji, H.-B.; Shi, H.-X. Chin. J. Org. Chem. 2014, 34, 630. (in Chinese)

(沈海民, 武宏科, 纪红兵, 史鸿金金, 有机化学, 2014, 34, 630.)

(e) Shen, H.-M.; Wu, H.-K.; Ji, H.-B.; Shi, H.-X. Chin. J. Org. Chem. 2014, 34, 1549 (in Chinese)

(沈海民, 纪红兵, 武宏科, 史鸿金金, 有机化学, 2014, 34, 1549.)

[6] Khan, A. R.; Forgo, P.; Stine, K. J.; D'Souza, V. T. Chem. Rev. 1998, 98, 1977.

[7] Breslow, R.; Canary, J. W.; Varney, M.; Waddell, S. T.; Yang, D. J. Am. Chem. Soc. 1990, 112, 5212.

[8] Liu, Y.; Zhao, Y.-L.; Chen, Y.; Liang, P.; Li, L. Tetrahedron Lett. 2005, 46, 2507

[9] (a) Sridhar, R.; Srinivas, B.; Madhav, B.; Reddy, V. P.; Nageswar, Y V. D.; Rao, K. R. Can. J. Chem. 2009, 87, 1704.

(b) Kumar, V. P.; Sridhar, R.; Srinivas, B.; Narender, M.; Rao, K. R. Can. J. Chem. 2008, 86, 907.

(c) Srinivas, B.; Kumar, V. P.; Sridhar, R.; Surendra, K.; Nageswar, Y. V. D.; Rao, K. R. J. Mol. Catal. A: Chem. 2007, 261, 1.

(d) Sridhar, R.; Srinivas, B.; Kumar, V. P.; Narender, M.; Rao, K. R. Adv. Synth. Catal. 2007, 349, 1873.

(e) Reddy, M. S.; Narender, M.; Nageswar, Y. V. D.; Rao, K. R. Synth. Commun. 2007, 37, 1983.

(f) Kumar, V. P.; Narender, M.; Sridhar, R.; Nageswar, Y. V. D.; Rao, K. R. Synth. Commun. 2007, 37, 4331.

(g) Surendra, K.; Krishnaveni, N. S.; Sridhar, R.; Rao, K. R. Tetrahedron Lett. 2006, 47, 2125.

(h) Surendra, K.; Krishnaveni, N. S.; Rao, K. R. Tetrahedron Lett 2006, 47, 2133.

(i) Krishnaveni, N. S.; Surendra, K.; Rao, K. R. Adv. Synth. Catal. 2006, 348, 696.

(j) Krishnaveni, N. S.; Surendra, K.; Rao, K. R. Chem. Commun. 2005, 669 .

[10] (a) Ramesh, K.; Karnakar, K.; Satish, G.; Anil Kumar, B. S. P.; Nageswar, Y. V. D. Tetrahedron Lett. 2012, 53, 6936.

(b) Madhav, B.; Narayana Murthy, S.; Anil Kumar, B. S. P.; Ramesh, K.; Nageswar, Y. V. D. Tetrahedron Lett. 2012, 53, 3835.

(c) Ramesh, K.; Narayana Murthy, S.; Karnakar, K.; Nageswar, Y. V. D. Tetrahedron Lett. 2011, 52, 4734.

(d) Ramesh, K.; Karnakar, K.; Satish, G.; Nageswar, Y. V. D. Chin Chem. Lett. 2012, 23, 1331.

[11] Kumar, A.; Tripathi, V. D.; Kumar, P. Green Chem. 2011, 13, 51.

[12] Shin, J. A.; Lim, Y. G.; Lee, K. H. J. Org. Chem. 2012, 77, 4117.

[13] Ji, H.-B.; Shi, D.-P.; Shao, M.; Li, Z.; Wang, L.-F. Tetrahedron Lett. 2005, 46, 2517.

[14] Patil, D. R.; Wagh, Y. B.; Ingole, P. G.; Singh, K.; Dalal, D. S. New J. Chem. 2013, 37, 3261 . 
[15] Liberto, N. A.; de Paiva Silva, S.; de Fátima, Â.; Fernandes, S. A. Tetrahedron 2013, 69, 8245.

[16] Breslow, R.; Duggan, P. J.; Light, J. P. J. Am. Chem. Soc. 1992, 114, 3982.

[17] Maksimov, A. L.; Sakharov, D. A.; Filippova, T. Y.; Zhuchkova, A. Y.; Karakhanov, E. A. Ind. Eng. Chem. Res. 2005, 44, 8644

[18] (a) Hapiot, F.; Bricout, H.; Tilloy, S.; Monflier, E. Eur. J. Inorg. Chem. 2012, 2012, 1571.

(b) Al-Maksoud, W.; Menuel, S.; Jahjah, M.; Monflier, E.; Pinel, C.; Djakovitch, L. Appl. Catal., A 2014, 469, 250.

(c) Six, N.; Guerriero, A.; Landy, D.; Peruzzini, M.; Gonsalvi, L.; Hapiot, F.; Monflier, E. Catal. Sci. Technol. 2011, 1, 1347.

(d) Leger, B.; Menuel, S.; Landy, D.; Blach, J. F.; Monflier, E.; Ponchel, A. Chem. Commun. 2010, 46, 7382.

[19] Guitet, M.; Marcelo, F.; de Beaumais, S. A.; Zhang, Y.; Jiménez-Barbero, J.; Tilloy, S.; Monflier, E.; Ménand, M.; Sollogoub, M. Eur. J. Org. Chem. 2013, 2013, 3691.

[20] Herbois, R.; Noel, S.; Leger, B.; Bai, L.; Roucoux, A.; Monflier, E.; Ponchel, A. Chem. Commun. 2012, 48, 3451.

[21] Tran, D. N.; Legrand, F. X.; Menuel, S.; Bricout, H.; Tilloy, S.; Monflier, E. Chem. Commun. 2012, 48, 753.

[22] Suresh, P.; Pitchumani, K. J. Org. Chem. 2008, 73, 9121.

[23] Azath, I. A.; Suresh, P.; Pitchumani, K. New J. Chem. 2012, 36, 2334.

[24] (a) Kaboudin, B.; Abedi, Y.; Yokomatsu, T. Eur. J. Org. Chem. 2011, 2011, 6656 .

(b) Kaboudin, B.; Abedi, Y.; Yokomatsu, T. Org. Biomol. Chem. 2012, 10, 4543 .

(c) Kaboudin, B.; Mostafalu, R.; Yokomatsu, T. Green Chem. 2013, $15,2266$.

[25] (a) Jouffroy, M.; Gramage-Doria, R.; Armspach, D.; Sémeril, D.; Oberhauser, W.; Matt, D.; Toupet, L. Angew. Chem., Int. Ed. 2014, $53,3937$.

(b) Jouffroy, M.; Sémeril, D.; Armspach, D.; Matt, D. Eur. J. Org. Chem. 2013, 2013, 6069.

(c) Gramage-Doria, R.; Armspach, D.; Matt, D.; Toupet, L. Dalton Trans 2012, 41, 8786.

[26] Hong S.; Liu M.; Yuan S.; Wang Z.; Shi L.; Deng W. J. Inclusion Phenom. Macrocyclic Chem. 2014, 80, 443.

[27] (a) Zhou, Y.; Pedersen, C. M.; Bols, M. Tetrahedron Lett. 2013, 54, 2458 .

(b) Zhou, Y.; Lindbäck, E.; Marinescu, L. G.; Pedersen, C. M.; Bols, M. Eur. J. Org. Chem. 2012, 2012, 4063. (c) Lindbäck, E.; Zhou, Y.; Pedersen, C. M.; Bols, M. Tetrahedron Lett. 2012, 53, 5023

[28] (a) Chen, H.; Ji, H. Supramol. Chem. 2014, 1.

(b) Yang, Z.; Ji, H. ACS Sustainable Chem. Eng. 2013, 1, 1172.

(c) Yang, Z.; Zeng, H.; Zhou, X.; Ji, H. Tetrahedron 2012, 68, 5912.

(d) Shen, H.-M.; Ji, H.-B. Tetrahedron Lett. 2012, 53, 3541.

(e) Long, Q.; Ji, H.; Lu, S. Comput. Theor. Chem. 2011, 963, 200.

[29] Azath, I. A.; Puthiaraj, P.; Pitchumani, K. ACS Sustainable Chem. Eng. 2012, 1, 174.

[30] Kanagaraj, K.; Pitchumani, K. Tetrahedron Lett. 2010, 51, 3312.

[31] Hu, S.; Li, J.; Xiang, J.; Pan, J.; Luo, S.; Cheng, J.-P. J. Am. Chem. Soc. 2010, 132, 7216 .

[32] Shen, H.-M.; Ji, H.-B. Tetrahedron 2013, 69, 8360.

[33] Guitet, M.; Zhang, P.; Marcelo, F.; Tugny, C.; Jimenez-Barbero, J.; Buriez, O.; Amatore, C.; Mouries-Mansuy, V.; Goddard, J. P.; Fensterbank, L.; Zhang, Y.; Roland, S.; Menand, M.; Sollogoub, M. Angew. Chem., Int. Ed. 2013, 52, 7213.

[34] Kanagaraj, K.; Suresh, P.; Pitchumani, K. Org. Lett. 2010, 12, 4070.

[35] Kanagaraj, K.; Pitchumani, K. J. Org. Chem. 2013, 78, 744.

[36] (a) Herrmann, W.; Wehrle, S.; Wenz, G. Chem. Commun. 1997, 1709.

(b) Rao, K. S. S. P.; Hubig, S. M.; Moorthy, J. N.; Kochi, J. K. J. Org. Chem. 1999, 64, 8098.

(c) Utsuki, T.; Hirayama, F.; Uekama, K. J. Chem. Soc., Perkin Trans. 2 1993, 109.

[37] Brinker, U. H.; Walla, P.; Krois, D.; Arion, V. B. Eur. J. Org. Chem. 2011, 2011, 1249.

[38] Marconi, G.; Mezzina, E.; Manet, I.; Manoli, F.; Zambelli, B.; Monti, S. Photochem. Photobiol. Sci. 2011, 10, 48.

[39] Ikeda, H.; Nihei, T.; Ueno, A. J. Org. Chem. 2005, 70, 1237.

[40] (a) Yang, C.; Wang, Q.; Yamauchi, M.; Yao, J.; Zhou, D.; Nishijima, M.; Fukuhara, G.; Mori, T.; Liu, Y.; Inoue, Y. Photochem. Photobiol. Sci. 2014, 13, 190 .

(b) Fukuhara, G.; Umehara, H.; Higashino, S.; Nishijima, M.; Yang, C.; Mori, T.; Wada, T.; Inoue, Y. Photochem. Photobiol. Sci. 2014, 13, 162 .

(c) Liang, W.; Yang, C.; Nishijima, M.; Fukuhara, G.; Mori, T.; Mele, A.; Castiglione, F.; Caldera, F.; Trotta, F.; Inoue, Y. Beilstein J. Org. Chem. 2012, 8, 1305.

(d) Wang, Q.; Yang, C.; Ke, C.; Fukuhara, G.; Mori, T.; Liu, Y.; Inoue, Y. Chem. Commun. 2011, 47, 6849.

[41] Yang, C.; Ke, C.; Liang, W.; Fukuhara, G.; Mori, T.; Liu, Y.; Inoue, Y. J. Am. Chem. Soc. 2011, 133, 13786. 